\section{DESARROLLO DEL DEBATE TEÓRICO EN TORNO AL NACIONALISMO Y LA NACIÓN A TRAVÉS DEL ESQUEMA KUHNIANO}

\author{
Guillermo Reyes Pascual \\ University of Kent \\ ORCID iD: https://orcid.org/0000-0002-7026-4242 \\ gr242@kent.ac.uk | guillepasc@gmail.com
}

\section{DEVELOPMENT OF THE THEORETICAL DEBATE REGARDING NATIONALISM AND THE NATION THROUGH THE KUHNIAN SCHEME}

Cómo citar este artículo/Citation: Reyes Pascual, G. (2019). Desarrollo del debate teórico en torno al nacionalismo y la nación a través del esquema kuhniano. Arbor, 195 (794): a532. https://doi.org/10.3989/arbor.2019.794n4006

Recibido: 28 enero 2019. Aceptado: 28 marzo 2019.

RESUMEN: Mucho se ha escrito para analizar y dar sentido a dos términos que vienen intrínsecamente unidos, el nacionalismo y la nación. La principal consecuencia positiva de esto ha sido un vasto debate teórico sobre el nacionalismo y la nación que indudablemente los enriquece. La principal consecuencia negativa, por su parte, es que el desarrollo del propio debate, y cómo se ha ido formando, ha recibido poca atención. El objetivo de este artículo es exponer cómo este debate se ha sido desarrollado alrededor de los cuatros paradigmas clásicos del nacionalismo. Para ello, se aplica el esquema kuhniano para saber cuándo, cómo, y por qué se articularon estos paradigmas clásicos en torno a estos dos términos. Con esto se pretende profundizar en la explicación histórica de Moreno Almendral (2015), pero desde la filosofía de la ciencia, desarrollando una visión mucho más detallada del debate en torno al nacionalismo y la nación.

PALABRAS CLAVE: Esquema kuhniano; nacionalismo; nación; revolución científica; paradigmas.
Copyright: (C) 2019 CSIC. Este es un artículo de acceso abierto distribuido bajo los términos de la licencia de uso y distribución Creative Commons Reconocimiento 4.0 Internacional (CC BY 4.0).
ABSTRACT: For purposes of analysis and also to find meaning, much has been written about two intrinsically-linked terms: nationalism and the nation. The main positive consequence of this has been a vast theoretical debate about nationalism and the nation that undoubtedly enriches them. On the other hand, the main negative consequence is that development of the debate itself and its evolution have received little attention. The aim of this article is to show how this debate was constructed around the four classical paradigms of nationalism. To do this, the Kuhnian scheme is applied to determine when, how, and why these classical paradigms were articulated around these two terms. The main purpose of this is to delve into the historical explanation given by Moreno Almendral (2015), but from the philosophy of science, developing a much more detailed vision of the debate around nationalism and the nation.

KEYWORDS: Kuhnian scheme; nationalism; nation; scientific revolution; paradigms. 


\section{INTRODUCCIÓN}

La doctrina dedica mucho espacio al debate en torno al nacionalismo y la nación. Por ejemplo, autores como Anthony Smith (2001), Philip Spencer y Howard Wollman (2005), Antonie Roger (2001) o Ronald Beiner (1999) han intentado compilar los aspectos más importantes de lo que la doctrina entiende como una de las principales controversias de la era contemporánea. Desde los diferentes paradigmas se intenta dar sentido a una de las fuerzas que legitiman el poder político desde las revoluciones liberales hasta la actualidad.

Una de las principales consecuencias es que el desarrollo del propio debate se ha dejado de lado, prácticamente como una nota histórica a pie de página, a pesar de que hay importantes autores que se han dedicado a su estudio, como Lawrence (2005) o Özkirimli (2010). El objetivo principal de este artículo es completar la explicación histórica del desarrollado del debate en torno al nacionalismo y la nación desarrollada por Moreno Almendral (2015), pero poniendo énfasis en entender qué fundamenta a estos diferentes paradigmas que han surgido a largo de dicho debate desde el punto de vista de la filosofía de las ciencias, acudiendo para ello a un clásico como es Tomas Kuhn (1970). Si el fenómeno que hay que estudiar es el nacionalismo y la nación, aquí se pone el foco en cuándo, cómo y por qué surgen determinados conjuntos de ideas o de saberes científicos en torno a este dilema, que son calificados como paradigmas clásicos del nacionalismo (Conversi, 2007): primordialismo, modernismo, perennialismo y etnosimbolismo. El propósito es arrojar luz sobre un aspecto del debate que normalmente atrae poca atención y que complementaría la doctrina en orden a una explicación más desarrollada sobre este dilema fundamental en las ciencias políticas.

Este artículo contiene tres epígrafes principales. El primero se refiere al marco teórico del esquema kuhniano, que explica el desarrollo científico articulado en torno a paradigmas. El segundo versa sobre el surgimiento y desarrollo del debate en torno al nacionalismo y la nación, poniendo el foco en un período histórico concreto. El tercero y último hace referencia a la aplicación del esquema kuhniano al debate en torno al nacionalismo y la nación para explicar cuándo, cómo y por qué surgen los diferentes paradigmas clásicos sobre dicho fenómeno.

\section{MARCO TEÓRICO DEL ESQUEMA KUHNIANO}

Tomando el rol de filosofo de las ciencias, el eje principal de la teoría kuhniana radica en la idea de que la ciencia, y el desarrollo de la misma, surge por contraposición y sustitución de un cuerpo doctrinal que explica un determinado fenómeno por otros cuerpos doctrinales que son capaces de dar solución a los problemas a los que los anteriores no han podido darla. Dicho cuerpo teórico es denominado por Kuhn (1970) como paradigma (p. 13), aunque es cierto que, en un principio, este término fue usado de forma ambivalente y poco consistente (HoyningenHuene,1993, pp. 131-132), con hasta veintiún posibles usos (Margret Masterman, 1970).

Estos paradigmas pueden ser definidos como un cuerpo de conocimiento científico aceptado por la mayoría de la comunidad científica y que da una explicación a los diferentes problemas que surgen del estudio de un determinado fenómeno (Wray, 2011, p. 387). Dos son los componentes principales. Por un lado, está el componente o matriz disciplinaria (Kuhn, 1970, pp. 10-13), que hace referencia a una narrativa científica que proporciona un modelo a seguir en la explicación de un determinado asunto científico y sus posibles problemas. Esta narrativa tiene que ser aceptada de forma genérica por la comunidad científica. Por otro lado, está el componente sociológico (Kuhn, 1970, p. 271), que hace referencia a que todos los miembros de dicha comunidad científica comparten esta narrativa como denominador común en el estudio del fenómeno. Así, un paradigma se caracteriza por un proceso de actividad científica basado en una fuerte red de compromisos conceptuales, teóricos, instrumentales y metodológicos por parte de una comunidad científica determinada y bien definida (Kuhn, 1970, p. 42).

El esquema kuhniano parte de lo que se denomina como ciencia normal, teniendo como característica principal el estudio de un fenómeno concreto a través de distintos procedimientos científicos donde las soluciones a los problemas que dicho fenómeno genera son convergentes (Firinci Orman, 2016, pp. 48-49), integrantes (Nagel, 1961, pp. 311-322) y acumulativas pero no lineales (Moulines, 2015, p. 73), para crear un paradigma científico que sea aceptado por la mayoría de la doctrina (Marcum, 2013, pp. 43-47). Dichos procedimientos científicos, o investigaciones normales, tienden a ser expansivos en el sentido de que van llenando los espacios vacíos que el estudio del fenómeno va creando a lo largo de su explicación. La ciencia normal se ayuda de estos procedimientos científicos 
para consolidar la vigencia del paradigma científico y refinar los distintos conceptos y modelos que explican el fenómeno (Buchwald y Smith, 1997, pp. 68-72). Un paradigma cumple tres funciones: la primara, sugiere nuevos posibles problemas que hay que solucionar dentro del paradigma; la segunda, proporciona posibles soluciones para resolverlos; y la tercera, es el estándar que se usa para medir la calidad de las posibles soluciones a los posibles problemas que han surgido o se han identificado (Kuhn, 1970, pp. 38-39).

A la ciencia normal puede que le siga un periodo al que se denomina como crisis de la ciencia normal (Kuhn, 1970, pp. 66-76). Tiene su origen en la imposibilidad de solucionar los posibles problemas que la ciencia normal identifica y que el paradigma vigente no puede resolver. La identificación y acumulación de problemas sin solución quebranta la validez de la explicación del fenómeno que el paradigma hasta ahora vigente proporciona (Bird, 2012, pp. 860-862). Así, la ciencia normal es sustituida por una revolución cien- tífica que es capaz de proporcionar nuevos conceptos y modelos que sí dan respuestas y soluciones, lo que tiene como principal consecuencia la aparición de un nuevo paradigma (Kuhn, 1970, pp. 77-110) que sustituye al viejo (Swerdlow, 2004, pp. 76-77).

El último punto que hay que discutir es la relación entre el paradigma descartado y el paradigma que va a ocupar el espacio del primero. Esa relación se denomina inconmensurabilidad de los paradigmas (Kuhn, 1970, pp. 66-67). Los paradigmas son inconmensurables cuando no comparten una explicación común al fenómeno que estudian (Malone, 1993, pp. 73-75). Si los paradigmas son los cuerpos de conocimientos científicos que dan soluciones a los problemas que surgen del estudio de un determinado fenómeno, estas mismas soluciones se juzgan siempre comparativamente (Hoyningen-Huene, 1990, pp. 490-492). El término inconmensurable deriva del hecho de que no hay una unidad de medida o narrativa común que pueda usarse para explicar un mismo fenómeno.

Figura 1. Explicación de la ciencia normal

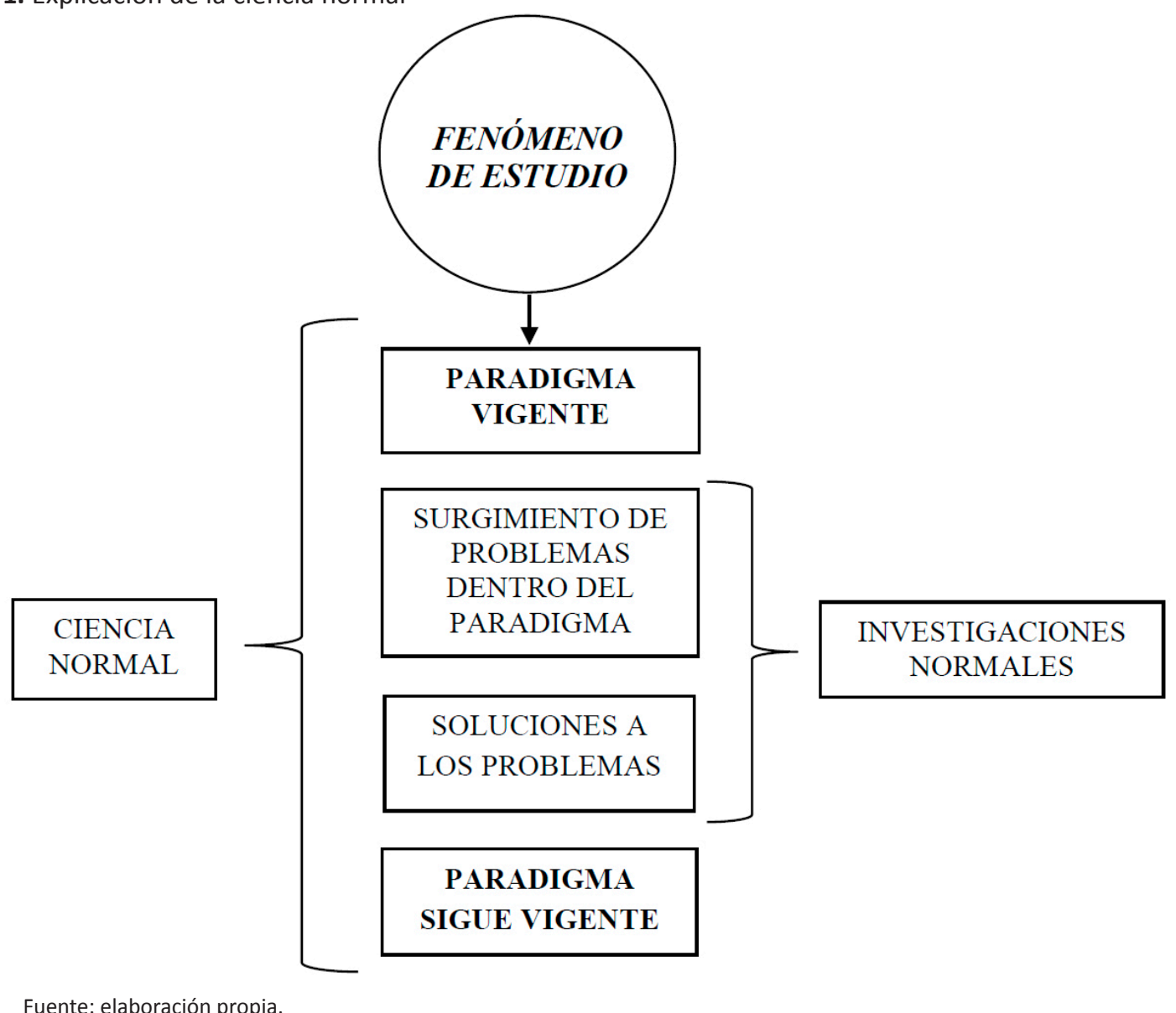

Fuente: elaboración propia. 
Figura 2. Revolución científica y sustitución de los paradigmas

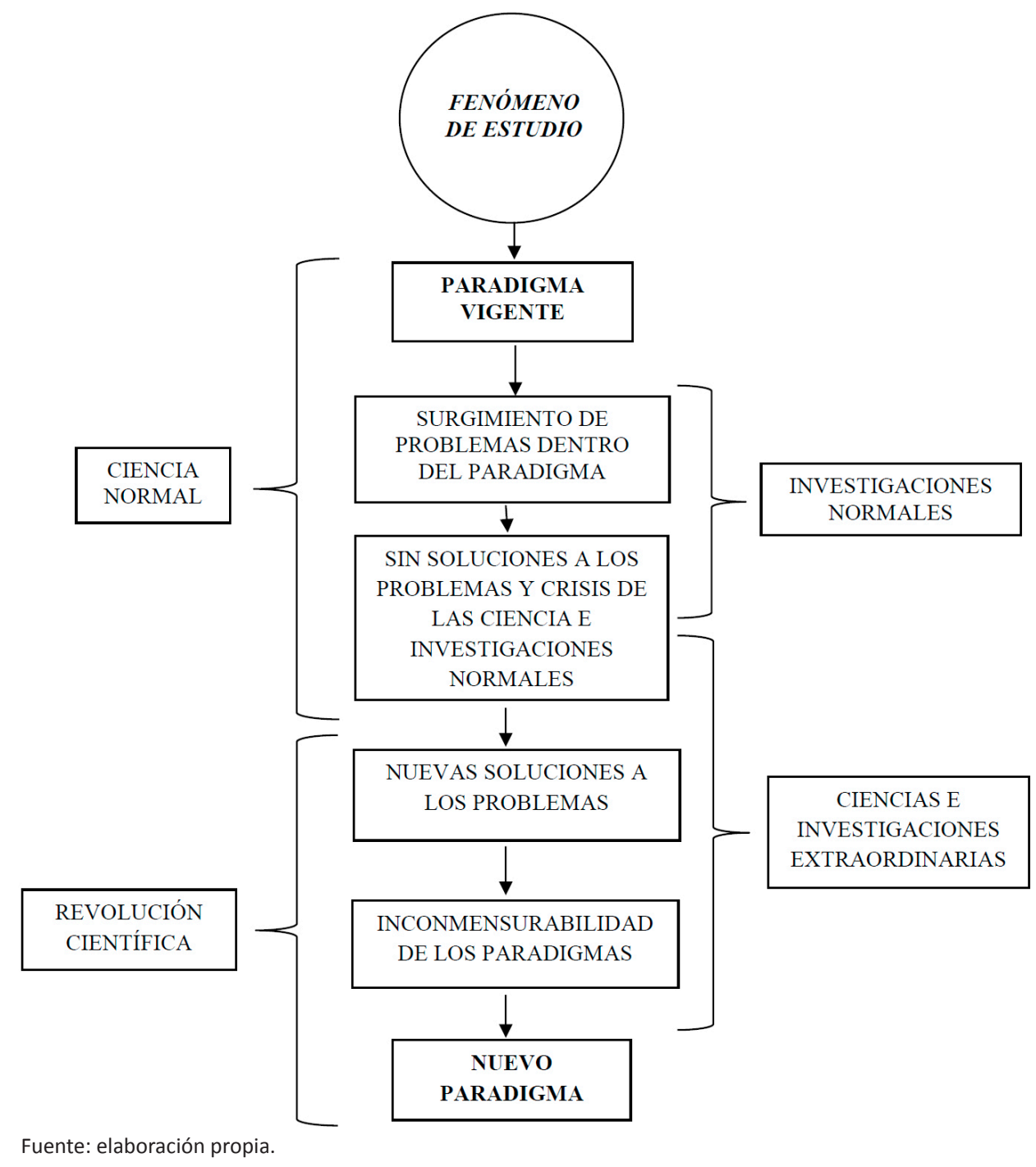

Se puede resumir el esquema kuhniano en dos fases principales. La primera fase toma en consideración la creación y consolidación de un paradigma que da una respuesta al estudio de un fenómeno. La comunidad científica, a través de la ciencia y la investigación normal, satisface la necesidad de abarcar todo aquello que el propio fenómeno genera. La segunda fase desarrolla la situación donde el paradigma vigente no da las necesarias respuestas a los problemas que surgen del estudio del fenómeno, y que la expansión del propio paradigma va identificando. El paradigma hasta ahora vigente pasa a ser ineficiente a la hora de dar respuestas. A través de una revolución científica, el paradigma ineficiente es sustituido por un paradigma nuevo y capaz de satisfacer las necesidades científicas en el estudio del fenómeno.

Entonces ¿qué puede identificar y diferenciar a un paradigma de otro? Un paradigma responde a los siguientes principios: ontológico, epistemológico y metodológico. El principio ontológico se ocupa de la naturaleza del fenómeno que se investiga (Poli, 2010, pp. 1-7), el principio epistemológico trata de la relación entre el fenómeno y el científico (Williams, 2001, pp. 1-12), ya sea esta relación objetiva, constructiva o subjetiva, por ejemplo (Biesta, 2010, pp. 110-115), y finalmente el principio metodológico alude a los procedimientos que el científico usa para analizar el fenómeno que estudia (Sarantakos, 2005, pp. 7). Estos 
principios deben ser entendidos y tomados en cuenta como entrelazados e inseparables puesto que la combinación de los tres da a los diferentes paradigmas su naturaleza particular y que, por tanto, los diferencia entre sí (Popkewitz, Tabacnick y Zeichner, 1979, p. 52). El paradigma es el cuerpo de conocimiento científico que contiene unas determinadas premisas epistemológicas, ontológicas y metodológicas (Denzin y Lincoln, 2000, p. 6). La combinación de estos principios es como la marca genética de cada paradigma, no hay dos iguales.

Por tanto ¿los paradigmas se diferencian entre sí porque todos enfocan cada uno de los tres principios de forma diferente, o pueden algunos paradigmas compartir el enfoque de alguno de los tres principios? Lo que la doctrina tiene claro es que cada paradigma tiene una combinación especifica de los tres principios que lo diferencian del resto, no que dos o más paradigmas no puedan compartir el mismo enfoque en alguno de los tres principios. El científico tiene libertad para estudiar el fenómeno de la forma que considere más adecuada (Cohen, Manion y Morrison, 2000, pp. 17-21), siendo esto clave para poder encontrar respuesta a dicho fenómeno, sin la cual esto último no sería posible. El científico puede articular un paradigma usando la combinación de estos tres principios que considere más adecuada. Lo importante es que esta combinación se convierta en piedra angular de la definición del paradigma, sea cual sea la combinación.

\section{SURGIMIENTO Y DESARROLLO DEL DEBATE EN TORNO AL NACIONALISMO Y LA NACIÓN}

Cuatro son los periodos principales en los que se puede dividir este debate. El primero comprende los siglos XVIII, XIX y principios del XX, donde se sientan las bases del cuerpo doctrinal del nacionalismo como ideología o movimiento político. El segundo corresponde al periodo comprendido entre el final de la Gran Guerra y el final de la Segunda Guerra Mundial, donde se inician los debates puramente académicos sobre el fenómeno. El tercero va desde el final de la Segunda Guerra Mundial hasta la década de 1990, y alumbra una revolución en la doctrina que se inicia con el cuestionamiento de las ideas preconcebidas sobre el nacionalismo y la nación. El cuarto y último periodo va desde finales del siglo XX hasta la actualidad, y tiene como principal hilo conductor el surgimiento de varias líneas de investigación que rompen con la anterior forma de estudiar dicha problemática.

Siguiendo a Moreno Almendral (2015), hay que resaltar que el periodo más importante es, sin duda, el tercero. Este periodo se puede explicar por las siguientes características: primero, el impacto que tuvo la Segunda Guerra Mundial en los escritos académicos de este periodo fue el mismo que el de la Gran Guerra; segundo, la creación de una comunidad académica alrededor del nacionalismo y la nación proporcionó el sustento definitivo para la interconectividad de varios campos del conocimiento; y tercero, en este periodo surgen los grandes paradigmas clásicos del nacionalismo que marcarán los debates hasta nuestros días.

Como primera característica, y al igual que tras la Primera Guerra Mundial, se puede decir que los escritos del periodo inmediatamente posterior a la Segunda Guerra Mundial sufrieron del mismo rechazo hacia el nacionalismo integral (Lawrence, 2005, p. 107). A esto contribuyó el hecho de que la mayoría de los escritos no fueron realizados por académicos sino por activistas, intelectuales o políticos que necesitaban de una nueva legitimidad tras los desastres de la contienda. La principal consecuencia positiva fue que tanto el nacionalismo como la nación fueron objeto de renovado interés por parte del mundo académico.

La segunda de las características es que este renovado interés proporcionó las bases necesarias para la creación de una comunidad científica integrada y articulada alrededor del nacionalismo y la nación, siendo esto ya una realidad objetiva en los años 50 y 60 (Smith, 2000a, p. 28). Esta tiene sus orígenes en los debates historiográficos y psicológicos del periodo de entreguerras (Finlayson, 1998, pp. 147-150), a los que se unieron otras ramas del conocimiento como la antropología y la sociología. Esto produjo la interconectividad de varios campos del conocimiento, reforzando el carácter académico y científico del estudio y de la propia comunidad científica (Synder, 1954, p. 12). En cuanto al desarrollo de los paradigmas clásicos del nacionalismo, esta comunidad científica e interdisciplinar tuvo dos consecuencias principales. La primera es que este periodo consolida el enfoque académico y científico a través de los diferentes paradigmas que surgieron (Lawrence, 2005, p. 132), y la segunda, que la interdisciplinariedad fomentó el debate entre los mismos (Özkirimli, 2010, p. 42).

Pasando a la última y más importante característica, en este periodo surgen los que se conocen como paradigmas clásicos del estudio del nacionalismo. Durante la década de 1950 y 1960, varios autores publicaron obras que fueron el germen de lo que sería conocido como el modernismo clásico, y que fue producto de la interdisciplinariedad del debate (Özkirimli, 2010, p. 42). Autores como Deutsch (1953), Kedourie (1960) o Gell- 
ner (1964) fueron los que más contribuyeron a ello, basándose en la teoría funcionalista (Smith, 2001, p. 66) de Weber y Durkheim (Lawrence, 2005, p. 134). Aunque las obras de estos autores significaron el comienzo del debate académico entre los diferentes paradigmas clásicos, no lo hicieron de forma inmediata (Day y Thompson, 2004, p. 8). La década de 1980 vio un resurgir del paradigma clásico del modernismo para darle un nuevo impulso frente a las críticas de las décadas anteriores. Autores como Hechter (1975), Nairn (1977), Hobsbawm y Ranger (1983) o Anderson (1991) fueron los que más contribuyeron a que el modernismo clásico se consolidase como la ortodoxia dominante dentro de la comunidad científica (Lawrence, 2005, p. 160).

Las primeras críticas surgieron de los más convencidos primordialistas en la década de 1950 y 1960 (con un pequeño resurgir en la década de 1970 y 1980), como fueron Shils (1957) y Geertz (1963), fundamentando el paradigma primordialista. En la década de 1970, Smith (1971) cuestionó las principales características modernistas y fue uno de los arietes más experimentados contra el dogma del modernismo clásico (Day y Thompson, 2004, p. 8). De estas críticas surgió lo que más adelante se denominó como el paradigma del perennialismo que, gracias a autores como Fishman (1972), Gillingham (1992) o Hastings (1997), desarrolló la crítica más destacada hacia la modernidad del nacionalismo y la nación. El etnosimbolismo fue el último de los paradigmas clásicos del nacionalismo que desarrollaron autores que habían sido anteriormente perennialistas convencidos, como Smith (1999), Armstrong (1982) o Hutchinson (1987), criticando tanto el modernismo del nacionalismo y la nación como la permanencia histórica de las naciones (Lawrence, 2005, p. 161). Este debate entre los cuatro principales paradigmas clásicos del nacionalismo dejó obsoletas gran parte de las teorías anteriores, convirtiendo este debate en uno de las más prolíferos de las ciencias sociales (Anderson, 1991, p. xii).

\section{APLICACIÓN DEL ESQUEMA KUHNIANO AL DEBATE EN TORNO AL NACIONALISMO Y LA NACIÓN}

En este epígrafe se pretende aplicar el esquema kuhniano al anterior relato histórico para comprender el cuándo, el cómo y el por qué surgieron los que Smith (2000a) considera como paradigmas clásicos del nacionalismo (pp. 2-4). Para Kuhn (1970), los paradigmas son, o proporcionan, un marco general dentro del cual se analizan los problemas, y se intentan resolver, de un determinado fenómeno y se intentan resolver (p. 10). Bajo un paradigma se desarrolla(n) una(s) teoría(s) que explica(n) las características de un determinado objeto de estudio. Al establecimiento de un determinado paradigma le sigue una fase que Kuhn denominó como ciencia normal, donde se lleva a cabo la aplicación del propio paradigma para resolver los posibles problemas o anomalías que pudieran surgir del estudio de un determinado fenómeno. El paradigma es válido siempre y cuando las anomalías que surjan se puedan resolver al aplicarlo pero ¿qué pasa cuando la aplicación del paradigma no resuelve las anomalías que surgen? Una vez que un paradigma falle a la hora de explicar las posibles anomalías que puedan surgir, entonces Kuhn establece el proceso de revolución científica o ciencia revolucionaria, donde surge un nuevo paradigma que sí es capaz de resolverlas. La ciencia avanza contraponiendo los diferentes paradigmas que van surgiendo.

En Smith (2000a) se pueden encontrar los cuatro paradigmas clásicos del nacionalismo (modernismo, perennialismo, primordialismo, y etnosimbolismo) que han surgido para explicar el nacionalismo y la nación (pp. 27-51). La última observación que se debe tener en cuenta en la aplicación del esquema kuhniano al debate en torno al nacionalismo y la nación es cómo se despliega el mismo. Esto quiere decir que hay diferentes etapas dentro de un campo de estudio que determina el surgimiento y consolidación de los diferentes paradigmas (Firinci Orman, 2016, p. 49). La ciencia se desarrolla en seis grandes etapas: la primera etapa puede ser denominada como etapa pre-paradigmática; la segunda etapa puede ser considerada como la etapa del paradigma originario; la tercera etapa puede ser la etapa de la ciencia normal; la cuarta etapa se desarrolla como la etapa de crisis de la ciencia normal; la quinta puede calificarse como la etapa de la revolución científica; y la sexta y última etapa puede ser etiquetada como la etapa de la inconmensurabilidad.

En cuanto al despliegue de estas etapas, la conclusión es la siguiente. Las primeras dos etapas se van a desplegar de forma lineal temporalmente hablando, las siguientes tres etapas se van a desplegar de forma coincidente y simultánea, y la última de forma lineal otra vez. Si las etapas tercera, cuarta y quinta son coincidentes y simultáneas en su despliegue temporal, ¿por qué se consideran como etapas diferenciadas? La respuesta es que las etapas no se diferencian y definen por su aspecto temporal, sino por su objetivo y función. Cada etapa tiene un objetivo y función determinados. El aspecto temporal es importante, pero no determina el objetivo y la función, sino al revés. Así, por ejemplo, la tercera etapa, donde se desarroIla la ciencia normal, tiene como objetivo resolver las 
posibles anomalías que surgen al aplicar el paradigma vigente y, por tanto, su función es constructiva. En cambio, la quinta etapa, la revolución científica, tiene como objetivo el desarrollo de un paradigma nuevo, y por tanto su función es la de presentar un nuevo paradigma que sea novedoso. Al término de la sexta etapa, las etapas tercera, cuarta y quinta se reinician.

Las características de la primera etapa, la pre-paradigmática, pueden ser las siguientes (Chalmers, 1982, pp. 90-92): primero, no hay un claro paradigma que explique el fenómeno que se trata de estudiar, y segundo, ni siquiera se puede decir que haya un campo de estudio diferenciado y reglado por el método científico que dé respuesta a las incógnitas que el fenómeno provoca. Todo lo que hay es una mezcla de teorías muy poco desarrolladas sobre el tema y que carecen de un marco teórico claro que determine el devenir del estudio sobre el fenómeno concreto (Kuhn, 1970, p. 160). Se podría decir que esta primera etapa del esquema kuhniano puede corresponder a los dos primeros periodos históricos del debate. Es decir, la etapa preparadigmática correspondería a los periodos que van desde el siglo XVIII hasta principios del silgo XX, y desde aquí hasta el final de la Segunda Guerra Mundial. Durante estos dos primeros periodos, la conclusión es que el debate carecía de una explicación puramente académica respecto al fenómeno por el muy escaso desarrollo de las propias ciencias sociales (Ross, 1993, pp. 81-83) y que, aunque solo al final del segundo periodo se puede observar el inicio de una aproximación científica reglada por el método científico al debate
(Smith, 1992, p. 62), sí que se puede atisbar un cierto interés académico respecto del tema por parte de los primeros científicos sociales como Durkheim o Weber (Smith, 1983, pp. 19-25). No se podría hablar todavía de paradigmas, de comunidad científica, de ciencia normal o de revolución científica.

Es en los dos últimos periodos, los que van desde el final de la Segunda Guerra Mundial hasta 1990 y desde la década de los años 90 hasta la actualidad, cuando se desarrollan las etapas segunda, tercera, cuarta, quinta y sexta del esquema kuhniano. Ya se puede hablar de paradigmas, de comunidad científica, de ciencia normal y de revolución científica. Es en estos dos periodos en los que se alcanza la madurez científica del debate. A partir del final de la Segunda Guerra Mundial, las etapas del esquema kuhniano se van a desarrollar de forma más acelerada. En las décadas de los años 50 y 60 es cuando se puede decir que es gracias a la convergencia de varios campos de estudio, como la psicología, la historiografía o la antropología (Özkirimli, 2010, p. 42), como el primer paradigma surge, apoyándose en teorías mucho más amplias que procedían de periodos históricos anteriores, como la teoría funcionalista (Fulcher y Scott, 1999, pp. 31-40). Con ello se cumple la segunda etapa en el desarrollo científico y se deja definido el paradigma originario. Tan pronto como este paradigma originario pasa a ocupar el primer plano del debate, las discusiones vagas y sin un objetivo claro y bien definido de la primera etapa pre-paradigmática cesan de existir (Kuhn, 1970, p. 17).

Figura 3. Despliegue del esquema kuhniano

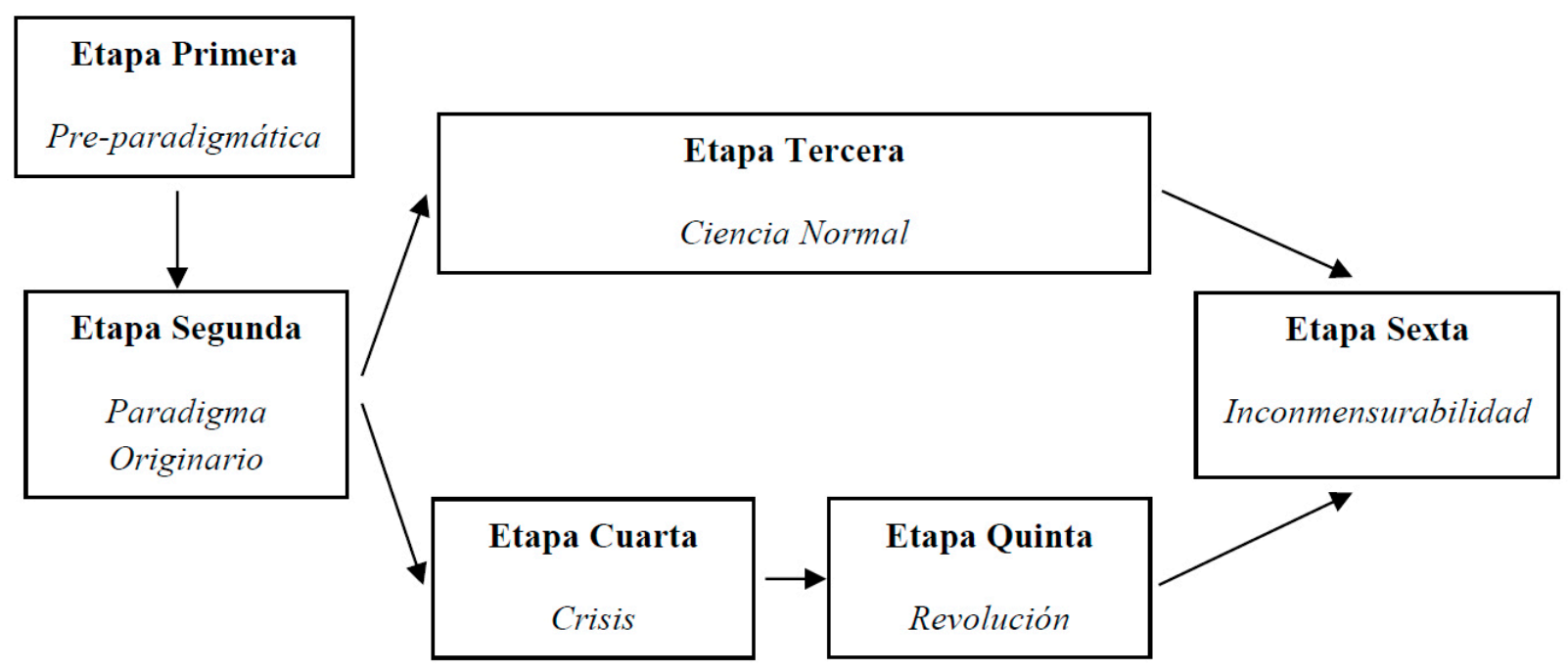

Fuente: elaboración propia. 
Dicho paradigma originario es el que se denomina como modernismo clásico (Smith, 2009, pp. 6-8) y el que define la segunda etapa del esquema kuhniano. Las características de este paradigma originario son las siguientes (Smith, 2000a, p. 21): el nacionalismo es producto de la modernidad y, por tanto, surge solo a partir de sus condiciones de ideología moderna; el nacionalismo y las naciones son construcciones sociales; las naciones son modernas, así como sus componentes básicos; las naciones son producto de la modernidad; y las naciones no tienen sus raíces enterradas en la historia, sino que son producto de la propia modernidad. Autores como Kedourie (1960), Gellner (1964), Worsley (1964) o Eisenstadt (1965) son algunos de los padres académicos de este paradigma originario. Todos provenían de campos académicos bien diferenciados (Smith, 2000a, p. 24), lo que supuso la simiente para la creación de una comunidad científica propia sobre el nacionalismo y la nación en la tercera etapa del esquema kuhniano. Ejemplos de esto último pueden ser los historiadores Hayes (1931), Kohn (1944) o Carr (1945), que introdujeron poco a poco una aproximación más científica y objetiva al estudio del nacionalismo y la nación (Palti, 2002, pp. 329-337). Carr introdujo dos novedades que los dos anteriores no consiguieron esclarecer. En primer lugar, estudió el nacionalismo y la nación de forma completamente objetiva, cosa que los dos anteriores historiadores no consiguieron del todo, posicionándose cerca del nacionalismo liberal anglosajón (Wolf, 1976, pp. 651-652) y, en segundo lugar, puso el foco en la novedad del nacionalismo y de la nación, cosa que Hayes o Kohn no hicieron (Breuilly, 2010, p. 1). Esto podría venir a confirmar que, por un lado, la primera etapa pre-paradigmática se caracterizó por la ausencia de un paradigma definido, pero no por la ausencia de posibles debates teóricos respecto al fenómeno y, por otro lado, que el surgimiento de un paradigma originario se sustentó sobre debates teóricos anteriores y no sobre la nada.

Lo anterior dio paso a la tercera etapa del esquema kuhniano, que se define por el desarrollo de la ciencia normal. Dos son las características claves. En primer lugar, el paradigma originario dio paso a una comunidad científica bien definida con derecho propio (Hoyningen-Huene, 1993, pp. 8-11). Esto es importante porque es esta misma comunidad la que va a desarrollar la ciencia normal en esta tercera etapa, y la que en la cuarta y quinta etapa intenta solventar la crisis de la ciencia normal y la posterior revolución científica (Marcum, 2005, pp. 60-61). Esto es un ejemplo claro de la madurez científica del debate. En segundo lugar, el paradigma originario también dio paso al desarrollo de la ciencia normal a través de la aplicación del paradigma del modernismo clásico, que explicaba el fenómeno y que contó con cierto consenso dentro de esta nueva comunidad científica (Kuhn, 1970, pp. 10-13 y p. 42).

La relación entre un paradigma y una teoría es un tanto difusa si uno acude a Kuhn, sobre todo en sus primeras reflexiones al respecto (Wray, 2011, pp. 387-388), por lo que hay que ir con sumo cuidado para explicar cómo se refinó el modernismo clásico para convertirse, a través de la ciencia normal, en uno de los paradigmas clásicos del nacionalismo. Para Vásquez (1998), la diferencia entre un paradigma y una teoría es la siguiente: en primer lugar, pueden existir varias teorías, y la sustitución de unas por otras dentro del mismo campo del conocimiento, sin que por ello haya un cambio de paradigma; y en segundo lugar, hay una relación recíproca entre el paradigma y las teorías, en el sentido de que el paradigma es previo a la existencia de estas teorías y, a su vez, estas últimas sustentan al primero (p. 22). Se podría decir que el paradigma es el marco dentro del cual se pueden encontrar varias teorías que son las que fundamentan al propio paradigma.

Cuando la aplicación del paradigma vigente -en este caso, el paradigma originario del modernismo clásico- choca con posibles anomalías en la explicación del fenómeno, las investigaciones normales dan como resultado diferentes teorías que dan solución a estas anomalías (Samian, 1994, p. 127), reforzando el propio paradigma (Kuhn, 1970, pp. 33-34), y es lo que se denomina como ciencia normal, siendo aceptada por la comunidad científica que lo aplica (Kuhn, 1970, p. 42). Acudiendo a Smith (2000a), se pueden encontrar cinco grandes teorías que pulieron el paradigma originario del modernismo clásico para definirlo como el actual paradigma clásico del modernismo (pp. 25-142). Estas teorías son: la teoría del industrialismo (Smith, 2000a, pp. 27-46), la teoría del capitalismo (Smith, 2000a, pp. 47-69), la teoría del estado y su influencia (Smith, 2000a, pp. 70-96), la teoría del mesianismo político (Smith, 2000a, pp. 97-116), y la teoría la de la invención del imaginario nacional (Smith, 2000a, pp. 117-142).

Autores como Gellner (1983) en el marco de la teoría del industrialismo, Kohn (1967), Hechter (1975) o Nairn (1977), dentro de las teorías del capitalismo, Tilly (1975) o Giddens (1985), dentro de las teorías del estado y su influencia, Kedourie (1971), en las teorías del mesianismo político, y Hobsbawm y 
Ranger (1983) o Anderson (1991), en las teorías de la invención del imaginario nacional, son los que, a través de sus específicas respuestas a las anomalías que el paradigma del modernismo clásico identificó, dieron forma al -ahora sí- paradigma clásico del modernismo. Ambos paradigmas no son el mismo per se, ya que el segundo es la evolución del primero. La consecuencia más destacable del desarrollo de la ciencia normal es precisamente la aparición de diferentes teorías dentro del propio paradigma originario del modernismo clásico, que lo refinó para convertirlo en uno de los cuatro: el paradigma clásico del nacionalismo, el paradigma clásico del modernismo, desarrollado en los años 60, 70, 80 y 90, y motor a su vez de la ciencia normal (Bird, 2012, p. 861). Este paradigma defiende que el nacionalismo es moderno cualitativa y cuantitativamente y que, cronológica y sociológicamente hablando, los nacionalismos y las naciones son fenómenos modernos, productos de las estructuras creadas por la era moderna.

A estas alturas es cuando la cuarta etapa, la crisis de la ciencia normal, entra en plena acción. La conclusión más importante al respecto es la siguiente: ciertas anomalías se utilizaron para desarrollar una serie de teorías que, gracias a su planteamiento novedoso, sirvieron para fundamentar, en la quinta etapa del esquema kuhniano, los restantes paradigmas clásicos del nacionalismo (Firinci Orman, 2016, p. 50). Tres son las anomalías que centran la discusión entre las teorías noveles de esta etapa (Smith, 2000 b, p. 2): la naturaleza del nacionalismo y la nación (Smith, 2000a, pp. 22-23), la antigüedad del nacionalismo y la nación (Özkirimli y Grosby,2007, pp. 523-529) y la función social del nacionalismo y la nación (Smith, 1995, pp. 3-8).

La aplicación de esta cuarta etapa dio como resultado las siguientes teorías que cuestionaron de frente el paradigma vigente en aquellos momentos, agrupadas en tres grandes grupos: el primer grupo está formado por las teorías que se encuentran bajo la lupa del nacionalismo académico, y que dan un lugar en la comunidad científica a los argumentos nacionalistas más básicos; el segundo grupo está formado por las teorías que, aunque tienen su punto de partida en el anterior grupo, se alejan de forma considerable de sus postulados más ideologizados; el tercer y último grupo está formado por las teorías que cuestionan a los grupos anteriores para distanciarse de los mismos y a su vez del paradigma clásico del modernismo.

En el primer grupo se pueden encontrar dos teorías destacables. La primera teoría se podría deno- minar como teoría culturalista. Las naciones y las comunidades étnicas se fundamentan sobre la lealtad y el afecto de los miembros a los elementos culturales de la propia comunidad (Smith, 2000a, p. 71). Los defensores de esta teoría, como Shils (1957) o Geertz (1963), sostienen que existen vínculos y lazos primarios originales que conviven con los vínculos y lazos civiles y seculares que existen en las sociedades modernas. Los vínculos y lazos que conviven con los lazos racionales de las naciones en la modernidad tienen las características esenciales de toda nación, como pueden ser la raza, la lengua, la religión, la tradición, el territorio, o el parentesco. Se pueden encontrar estos vínculos y lazos primarios en todas las naciones. La segunda teoría se puede denominar como teoría socio-biológica. Las comunidades que comparten una descendencia y un ancestro común lo hacen por lazos genéticos. El comportamiento social de la comunidad gira en torno a una mejora genética de las próximas generaciones (Berghe, 1978, p. 405). Las comunidades tienen como objetivo la extensión de su patrimonio genético más allá de sus propios lazos de parentesco. Una comunidad no es más que la red de parentesco por afinidad genética que comparte mitos y símbolos como marcas biogenéticas (Smith, 2000a, p. 70). Hay una relación intrínseca entre los mitos sobre los orígenes étnicos y los orígenes biológicos (Berghe, 1988, p. 256).

En el segundo grupo se pueden diferenciar dos grandes teorías (Smith, 2000a, p. 68). La primera teoría, defendida por autores como Greenfeld (1992) o Hastings (1997), se podría denominar como perennialismo continuo (Smith, 1999, pp. 5-27) y hace referencia a que las naciones anteceden a la modernidad. El rastro de la historia de esas naciones se puede encontrar en cualquier etapa histórica, desde la Antigüedad hasta la Edad Media, siendo esta continua y larga, aunque tenga un comienzo y un final. La identidad cultural de las naciones desarrolla su evolución a lo largo de un periodo continuo (Smith, 2000a, p. 168). La segunda teoría, defendida por autores como Fishman (1972) o Roschwald (2006), se podría denominar como perennialismo recurrente (Smith, 2000a, pp. 34-41), denominación que se refiere a que lo que es continuo es la idea de nación y no su forma concreta. Puede que se desarrolle dentro de un imperio o un virreinato $y$, aunque estas mismas formas cambien, lo que no cambia es la idea de nación. Dicha idea, de una forma u otra, siempre permanece (Smith, 2000a, p. 168). La recurrencia de la nación subyace en el ideal de nación. Puede que las formas de estado o de organización política cambien, pero no el ideal de nación. 
En el tercer y último grupo se pueden distinguir tres teorías principales (Maleševic, S. y Pavasovic Trošt, 2018). La primera de las teorías se podría denominar como teoría historicista. Esta teoría defiende los orígenes étnicos de las naciones, y está representada por autores como Smith (1986) o Hutchinson (1987). Se destaca la antigüedad de la nación sustentada sobre un parentesco común, así como una afinidad cultural con un pasado remoto en el que la propia comunidad cree, principal fuente de su propia legitimidad. Las instituciones creadas por la comunidad tienen como objetivo el de preservar sus propios repertorios culturales pre-modernos, que están formados por símbolos, mitos, y recuerdos de la misma. La segunda teoría se puede definir como teoría fenomenológica, que sostiene que las identidades étnicas deberían ser investigadas a lo largo de un amplio plazo o longue durée (Smith, 2000a, p. 196). Aquí se pueden encontrar autores como Armstrong (1982). La tercera y última teoría se podría denominar teoría psicológica de la comunidad (Yack, 2012), que defiende que las naciones solo pueden explicarse por las relaciones de mutuo interés y lealtad entre los diferentes grupos que las integran. Los grupos son capaces de imaginarse estas relaciones basadas en una cultura compartida.

La quinta etapa de la revolución científica se explica muy gráficamente con el surgimiento de los demás paradigmas clásicos del nacionalismo (Smith, 2009, pp. 3-22) a través de los tres grupos de teorías desarrolladas en la cuarta etapa. Las teorías desarrolladas por la aplicación de la ciencia normal de la tercera etapa, que dio como consecuencia el refinamiento del paradigma originario del modernismo clásico hacia el paradigma clásico del modernismo, no pueden considerarse incluidas en esta etapa porque no son producto de la crisis del paradigma originario, sino precisamente de su aplicación y posterior desarrollo. El primer grupo de teorías de la cuarta etapa, desarrolladas en los años 50, 60 y 70, sirvió para desarrollar el paradigma clásico del primordialismo (Smitn, 2000a, p. 146). Este paradigma se puede resumir en que las naciones son entidades orgánicas atemporales con características esenciales propias, y que el nacionalismo no es más que la expresión de pertenencia a una nación, fundamentado en las características esenciales y en los elementos diferenciadores. El segundo grupo de teorías de la cuarta etapa, desarrolladas en los años 70,80 , y 90 , sirvió para que el paradigma clásico del perennialismo tomara forma. Este paradigma defiende que hay naciones antiguas, que preexisten a la modernidad, y naciones modernas, y que el nacionalismo no es el único culpable de la creación de las naciones, sobre todo de las naciones antiguas. Por último, el tercer grupo de teorías de la cuarta etapa, desarrolladas en los años 80 y 90, fundamentó el último y más reciente de los paradigmas clásicos del nacionalismo, el etnosimbolismo, que defiende que se puede hacer una división entre naciones antiguas y modernas, y que en las últimas este sentimiento de pertenencia hacia las mismas no se construyó hasta la propia modernidad.

Cronológicamente hablando, y tomando la explicación de las etapas tercera, cuarta y quinta del esquema kuhniano, los diferentes paradigmas clásicos del nacionalismo surgieron de la siguiente manera. El primer paradigma clásico que surgió fue el primordialismo, que se enfrentó al paradigma originario del modernismo clásico. El modernismo clásico, a su vez, se refinó entonces para desarrollar el segundo de los paradigmas clásicos, el modernismo, para responder a las críticas del anterior (Smith, 2000a, p. 159). Luego sobrevino el tercer paradigma clásico, el perennialismo, que criticaba abiertamente a los dos anteriores, seguido por el paradigma clásico del etnosimbolismo (Smith, 2009, pp. 13-14), que es actualmente el último y más novedoso de ellos, siendo el que más desarrollo teórico está propiciando dentro de la doctrina.

La sexta y última etapa, la de la inconmensurabilidad, se va a desarrollar de forma lineal con respecto a las tres etapas anteriores. Esta etapa es incompatible con el desarrollo de cualquiera de las anteriores en el sentido de que la sustitución de un paradigma por otro anula el desarrollo de cualquier ciencia normal, crisis o revolución científica hasta que esta etapa de inconmensurabilidad haya finalizado. En un principio, en esta etapa es cuando uno de los paradigmas nuevos surgidos de la quinta etapa, la revolución científica, tiene la intención de sustituir al paradigma vigente y ser aceptado ampliamente por la mayoría de la comunidad científica creada durante la segunda etapa del paradigma originario. Así, el paradigma clásico del nacionalismo que está vigente desde la segunda etapa kuhniana, y que nunca ha dejado de estarlo desde que en los años 60 se refinó el paradigma originario del modernismo clásico (Smith, 2000a, p. 24), durante la tercera etapa es el paradigma clásico del modernismo (Conversi, 2007, p. 18). Su éxito radica en que ha sido aceptado de forma generalizada por la comunidad científica que estudia el nacionalismo y la nación (Smith, 2000a, p. 145). Por tanto, si solo ha sido aceptado un paradigma desde la segunda etapa kuhniana, ¿'eso significa que la sexta etapa de la inconmensurabilidad no se ha dado todavía? 
La respuesta seria que esta sexta etapa sí que se ha dado porque se tendría que entender la inconmensurabilidad de forma más amplia. La propia existencia y aceptación de diferentes paradigmas clásicos del nacionalismo por parte de la doctrina es la prueba más sólida de que la inconmensurabilidad no solo tiene que ver con la sustitución de un paradigma viejo e ineficaz por uno nuevo y más desarrollado, sino también por la vigencia y validez de uno ya existente frente a los demás paradigmas que pretenden ocupar su espacio. A su vez, esto también demostraría que las etapas tercera, cuarta y quinta pueden desarrollarse de forma simultánea y coincidente en su aspecto temporal, sin que por ello se anulen unas a otras. La última etapa, la de la inconmensurabilidad, es la que otra vez se relaciona de forma lineal con las tres anteriores. A partir de la vigencia de un paradigma (nuevo o no) las etapas tercera, cuarta y quinta vuelven a iniciarse al finalizar esta sexta etapa.

\section{CONCLUSIONES}

En este artículo se ha intentado completar la explicación histórica del desarrollo del debate en torno al nacionalismo y la nación (Moreno Almendral, 2015) desde el punto de vista de la filosofía de las ciencias. El esquema kuhniano ha servido para ver cuándo, cómo y por qué han surgido lo que la doctrina entiende como paradigmas clásicos del nacionalismo. Aplicando las seis etapas en las que se puede dividir este esquema, las principales conclusiones son las siguientes.
En cuanto al cuándo se desarrollaron los cuatro paradigmas clásicos del nacionalismo, se tiene que concluir que es en el periodo que va desde el final de la Segunda Guerra Mundial hasta los años 90 cuando se desarrollaron de forma clara el primordialismo, el modernismo, el perennialismo, y el etnosimbolismo. Antes de la Segunda Guerra Mundial se desarrolló lo que podría definirse como la etapa pre-paradigmática, donde no se puede hablar todavía de la existencia de posibles paradigmas, de comunidad científica, de ciencia normal, de revolución científica o de inconmensurabilidad de los paradigmas. Poniendo el foco en el cómo se desarrollaron, la deducción más importante es que es en las etapas tercera, cuarta, quinta y sexta del esquema kuhniano cuando se ve cómo, a través de la aplicación de la ciencia normal, la existencia de una crisis de la anterior, la revolución científica, y finalmente la inconmensurabilidad de un determinado paradigma, el modernismo (el clásico y su posterior evolución), el primordialismo, el perennialismo y el etnosimbolismo se cimentaron como los cuatro paradigmas clásicos del nacionalismo. Aunque hay una dialéctica académica muy productiva entre estos cuatro paradigmas, solo el modernismo ha tenido un éxito incuestionable en cuanto a su capacidad de explicar el nacionalismo y la nación. Finalmente, con respecto al por qué, son las incógnitas de la naturaleza del nacionalismo y la nación, de la antigüedad del nacionalismo y la nación y de la función social del nacionalismo y la nación las que sirven de sustento a estos cuatro paradigmas clásicos del nacionalismo, cada uno con una explicación propia.

\section{BIBLIOGRAFÍA}

Anderson, B. (1991). Imagined Communities. London: Verso.

Armstrong, J. (1982). Nations before Nationalism. Chapel Hill, North Carolina: University of North Carolina Press.

Beiner, R. (1999). Theorizing Nationalism. Albany: State University of New York.

Berghe, P. van der (1978). Race and ethnicity: a sociobiological perspective. Ethnic and Racial Studies, 1 (4), pp. 401-411. https://doi.org/10.1080/01419870.197 8.9993241

Berghe, P. van der (1988). Ethnicity and the sociobiology debate. En: Rex, J. y Mason, D. (eds.), Theories of Ethnic and Race Relations. Cambridge: Cambridge University Press, pp. 246-263. https://doi. org/10.1017/СВO9780511557828.013
Biesta, G. (2010). Pragmatism and the philosophical foundations of mixed methods research. En: Taskakkori, A. y Teddlie, C. (eds.). Handbook of Mixed Methods in Social and Behavioural Research. Thousand Oaks: Sage, pp. 95-118. https:// doi.org/10.4135/9781506335193.n4

Bird, A. (2012). The Structure of Scientific Revolutions and its Significance: An Essay Review of the Fiftieth Anniversary Edition. The British Journal for the Philosophy of Science, 63 (4), pp. 859-883. https://doi.org/10.1093/bjps/axs031

Breuilly, J. (2010). Bringing History Back into Nationalism. En: Aktar, A., Kizilyürek, N. y Özkirimli, U. (eds.). Nationalism in the Troubled Triangle. London: Palgrave MacMillan, pp. 1-20. https://doi. org/10.1057/9780230297326_1
Buchwald, J. Z. y Smith, G. E. (1997). Thomas S. Kuhn, 1922-1996. Philosophy of Science, 64 (2), pp. 361-376. https://doi. org/10.1086/392557

Carr, E. (1945). Nationalism and After. London: MacMillan.

Chalmers, A. (1982). What is This Thing Called Science? St. Lucia: University of Queensland Press.

Cohen, L., Manion, L. y Morrison, K. (2000). Research Methods in Education. London: Routledge Falmer.

Conversi, D. (2007). Mapping the Field Theories of Nationalism and the Ethnosymbolic Approach. En: Leoussi, A. S. y Grosby, S. (eds.). Nationalism and Ethnosymbolism. Edinburgh: Edinburgh University Press, pp. 15-30. 
Day, G. y Thompson, A. (2004). Theorizing Nationalism. Basingstoke: Palgrave MacMillan.

Denzin, N. K y Lincoln, Y. (2000). Introduction: The Discipline and Practice of Qualitative Research. En: Denzin, N. K. y Lincoln, Y. (eds.). Handbook of Qualitative Research: Thousand Oaks: Sage, pp. 1-29.

Deutsch, K. W. (1953). Nationalism and Social Communication. Cambridge, Massachusetts: MIT Press.

Eisenstadt, S. (1965). Modernisation: Protest and Change. Englewood Cliffs, New Jersey: Prentice-Hall.

Finlayson, A. (1998). Psychology, Psychoanalysis, and Theories of Nationalism. Nations and Nationalism, 4 (2), pp. 145 162. https://doi.org/10.1111/j.13545078.1998.00145.x

Firinci Orman, T. (2016). Paradigms as a Central Concept in Thomas Kuhn's Thought. International Journal of Humanities and Social Science, 6 (10), pp. 47-52.

Fishman, J. (1972). Language and Nationalism: Two Integrative Essays. Rowley, Massachusetts: Newbury House Publishers.

Fulcher, J. y Scott, J. (1999). Sociology. Oxford: Oxford University Press.

Geertz, C. (1963). Old Societies and New States. New Yok: Free Press.

Gellner, E. (1964). Thought and Change. London: Weidenfeld \& Nicolson.

Gellner, E. (1983). Nations and Nationalism. Oxford: Blackwell.

Giddens, A. (1985). The Nation-State and Violence. Cambridge: Cambridge University Press.

Gillingham, J. (1992). The Beginning of English Imperialism. Journal of Historical Sociology, 5, pp. 392-409. https:// doi.org/10.1111/j.1467-6443.1992. tb00033.x

Greenfeld, L. (1992). Nationalism: Five Roads to Modernity. Cambridge, Massachusetts: Harvard University Press.

Hastings, A. (1997). The Construction of $\mathrm{Na}$ tionhood. Cambridge: Cambridge University Press. https://doi.org/10.1017/ CB09780511612107

Hayes, C. (1931). The Historical Evolution of Modern Nationalism. Nueva York: Smith.

Hechter, M. (1975). Internal Colonialism: The Celtic Fringe in British National De- velopment, 1536-1966. London: Routledge and Kegan Paul.

Hobsbawm, E. y Ranger, T. (1983). The Invention of Tradition. Cambridge: Cambridge University Pres.

Hoyningen-Huene, P. (1990) Kuhn's Conception of Incommensurability. Studies in History and Philosophy of Science, 21 (3), pp. 481-492. https://doi. org/10.1016/0039-3681(90)90006-T

Hoyningen-Huene, P. (1993). Reconstructing Scientific Revolutions: Thomas S. Kuhn's Philosophy of Science. Chicago: University of Chicago Press. https://doi. org/10.1119/1.17343

Hutchinson, J. (1987). The Dynamics of Cultural Nationalism. London: Routledge.

Kedourie, E. (1960). Nationalism. Oxford: Blackwell.

Kedourie, E. (1971). Nationalism in Asia and Africa. Oxford: Weidenfeld and Nicolson.

Kohn, H. (1944). The Idea of Nationalism: A Study in its Origin and Background. Nueva York: The MacMillan Company.

Kohn, H. (1967). Prelude to Nation-State. Princeton, New Jersey: D. van Nostrand Company.

Kuhn, T. (1970). The Structure of Scientific Revolutions. Chicago: University of Chicago Press.

Lawrence, P. (2005). Nationalism. History and Theory. Edinburgh: Pearson Longman.

Maleševic, S. y Pavasovic Trošt, T. (2018). Nation-State and Nationalism. En: Ritzer, G. y Rojek, C. (eds.). Wiley Blackwell Encyclopedia of Sociology. London: Wiley Blackwell. https:// doi.org/10.1002/9781405165518. wbeosn003.pub2

Malone, M. (1993). Kuhn Reconstructed: Incommensurability without Relativism. Studies in History and Philosophy of Science, 24 (1), pp. 6993. https://doi.org/10.1016/00393681(93)90025-F

Marcum, J. A. (2005). Thomas Kuhn's RevoIution. London: Continuum.

Marcum, J. A. (2013). From Paradigm to Disciplinary Matrix and Exemplar. En: Kindi, V. y Arabatzis, T. (eds.). Kuhn's The Structure of Scientific Revolutions Revisited. London: Routledge, pp. 41-63.
Masterman, M. (1970). The Nature of a Paradigm. En: Lakatos, I. Musgrave, A. (eds.). Criticism and the Growth of Knowledge. Cambridge: Cambridge University Press, pp. 59-89. https://doi. org/10.1017/CBO9781139171434.008

Moreno Almendral, R. (2015). El debate académico sobre nación y nacionalismo desde los orígenes hasta la consolidación del predominio anglosajón. Arbor, 191 (775): a272. https://doi. org/10.3989/arbor.2015.775n5011

Moulines, C. U. (2015). Popper y Kuhn. Dos gigantes de la filosofía de la ciencia del Siglo XX. Madrid: Batiscafo.

Nagel. E. (1961). The structure of Science: Problems in the Logic of Scientific Explanation. London: Routledge and Kegan Paul.

Nairn, T. (1977). The Break-up of Britain: Crisis and Neo-Nationalism. London: New Left Books.

Özkirimli, U. (2010). Theories of Nationalism. Basingstoke: Palgrave MacMillan.

Özkirimli, U. y Grosby, S. (2007). Nationalism Theory Debate: The Antiquity of Nations? Nations and Nationalism, 13, pp. 523-537. https://doi.org/10.1111/ j.1469-8129.2007.00297.x

Palti, E. J. (2002). The Nation as a Problem. Historians and the National Question. History and Theory, 40 (3), pp. 324346. https://doi.org/10.1111/00182656.00171

Poli, R. (2010). Ontology: The Categorical Stance. En: Poli, R. Seibt, J. (eds.). Theory and Application of Ontology: Philosophical Perspectives. New York: Springer, pp. 1-22. https://doi.org/10.1007/97890-481-8845-1_1

Popkewitz, S. T., Tabacnick, B. R. yZeichner, K. M. (1979). Dulling the Senses: Research in Teacher Education. Journal of Teacher Education, 30 (5), pp. 52-60. https://doi. org/10.1177/002248717903000527

Roger, A. (2001). Les Grandes Théories du Nationalisme. Paris: Dalloz-Sirey.

Roschwald, A. (2006). The Endurance of Nationalism: Ancient Roots and Modern Dilemmas. Cambridge: Cambridge University Press.

Ross, D. (1993). The Development of the Social Sciences. En: Farr, J. y Seidelman, R. (eds.). Discipline and History. Political Science in the United States. Chicago: The University of Michigan Press, pp. 81-106. 
Samian, A. L. (1994). The Impact of Kuhn's Structure of Scientific Revolutions on Sociology of Science. Akademika, 44 (1), pp. 125-136.

Sarantakos, S. (2005). Social Research. Melbourne: Macmillan Education.

Shils, E. (1957). Primordial, Personal, Sacred, and Civil Ties. British Journal of Sociology, 8 (2), pp. 130-145. https://doi. org/10.2307/587365

Smith, A. (1971). Theories of Nationalism. London: Duckworth.

Smith, A. (1983). Nationalism and Classical Social Theory. The British Journal of Sociology, 34 (1), pp. 19-38. https://doi. org/10.2307/590606

Smith, A. (1986). The Ethnic Origins of $\mathrm{Na}$ tions. Oxford: Blackwell.

Smith, A. (1992). Nationalism and the Historians. International Journal of Comparative Sociology, XXXIII (1-2), pp. 58-80.

Smith, A. (1995). Gastronomy or Geology? The Role of Nationalism in the Reconstruction of Nations. Nations and Nationalism, 1, pp. 3-23.
Smith, A. (1999). Myths and Memories of the Nation. Oxford: Oxford University Press.

Smith, A. (2000a). Nationalism and Modernism. London: Routledge.

Smith, A. (2000b). The Nation in History: Historiographical Debates about Ethnicity and Nationalism. Hanover: University Press of New England.

Smith, A. (2001). Nationalism: Theory, Ideology, History. Cambridge: Polity Press.

Smith, A. (2009). Ethno-symbolism and $\mathrm{Na}$ tionalism. London: Routledge. https:// doi.org/10.4324/9780203876558

Spencer, P. y Wollman, H. (2005). Nations and Nationalism. Edinburgh: Edinburgh University Press.

Swerdlow, M. N. (2004). An Essay on Thomas Kuhn's First Scientific Revolution, The Copernican Revolution. Proceedings of the American Philosophical Society, 148 (1), pp. 64-120.

Synder, L. L. (1954). Meaning of Nationalism. New Jersey: Rutgers University Press.
Tilly, C. (1975). The Formation of National States in Western Europe. Princeton, New Jersey: Princeton University Press.

Vásquez, J. A. (1998). The Politics of Power: From Classical Realism to Neotraditionalism. Cambridge: Cambridge University Press.

Williams, M. J. (2001). Problems of Knowledge: A Critical Introduction to Epistemology. New York: Oxford University Press.

Wolf, K. (1976). Hans Kohn's Liberal Nationalism: The Historian as Prophet. Journal of the History of Ideas, 37 (4), pp. 651672. https://doi.org/10.2307/2709029

Worsley, P. (1964). The Third World. London: Weidenfeld and Nicolson.

Wray, K. B. (2011). Kuhn and the Discovery of Paradigms. Philosophy of the Social Sciences, 41 (3), pp. 380-397. https:// doi.org/10.1177/0048393109359778

Yack, B. (2012). Nationalism and the Moral Psychology of Community. Chicago: University of Chicago Press. https://doi.org/10.7208/chicago/9780226944685.001.0001 